\title{
Significados construidos por académicos acerca de la enseñanza de la lingüística para profesores en formación: un estudio de caso*
}

\author{
Meanings constructed by academics about the teaching of \\ linguistics for teacher training: a case study
}

Patricio Moya Muñoz ${ }^{* * *}$ Manuel Rubio Manríquez ${ }^{* * *}$

\section{RESUMEN}

En este artículo se describen los significados construidos por académicos del área de lingüística de una universidad respecto de la enseñanza de la disciplina a futuros profesores de L1. Corresponde a un estudio de caso cualitativo que implicó una entrevista a 11 académicos que enseñan en la carrera de Pedagogía en Castellano. Los resultados indican que el discurso de los académicos se distribuye en dos polos vinculados con la enseñanza de la lingüística: a) un saber científico válido en sí mismo y b) la formación de un sujeto activo consciente de sus prácticas discursivas. Se concluye que existe una tensión no abordada entre una visión aplicacionista de raigambre positivista versus una visión de reelaboración didáctica del contenido basada en un enfoque humanista.

\section{SUMMARY}

This article describes the meanings constructed by university academics that study linguistics in regard to the teaching of the discipline to future $\mathrm{L} 1$ teachers. It refers to a qualitative case study that involved interviewing 11 academics who teach degrees in Spanish Pedagogy. The results indicate that the discourse of academics is distributed into two areas linked to the teaching of
Palabras clave: consciencia lingüística, educación lingüística, formación inicial docente, prácticas discursivas.

Keywords: linguistic awareness, linguistic education, initial teacher training,

\footnotetext{
* Este trabajo forma parte del Proyecto POSTDOC-DICYT, Código 031951RM_ POSTDOC de la Universidad de Santiago de Chile, Usach.

** Doctor en Lenguas y Tecnología. Académico de la Universidad de Santiago de Chile, Usach. Santiago, Chile.patricio.moya@usach.cl.

*** Doctor en Lingüística. Académico de la Universidad de Santiago de Chile, Usach. Santiago, Chile.manuel.rubio@usach.cl.
} 
Significados construidos por académicos acerca de la enseñanza de la lingüística | Moya y Rubio

linguistics: a) a scientific knowledge valid in itself and b) the formation of an active subject aware of their discursive practices. The article concludes that there is an unaddressed tension between an applicationist vision of positivist roots versus a vision of didactic reworking of content based on a humanist approach. discursive practices. 


\section{Introducción}

En Chile, la formación inicial docente se ha constituido en un eje fundamental de las políticas públicas orientadas al mejoramiento de la calidad del sistema escolar, en particular considerando el incremento de programas de formación de profesores y la falta de regulación que ha caracterizado el sistema universitario chileno (Agencia de la Calidad de la Educación, 2016). Las críticas a la calidad de dichos programas no solo provienen de instancia gubernamentales, sino también de los profesores egresados, quienes han puesto especial énfasis en la debilidad en su formación didáctica (Gaete et al., 2016). En otras palabras, los profesores en formación abordan conocimientos de carácter disciplinar (lingüística y literatura en el caso de los profesores de L1) y una aproximación general al saber pedagógico, contando con pocas posibilidades para la reelaboración didáctica de dichos saberes disciplinares en el espacio escolar (Bahamondes et al., 2021). Dicha reelaboración didáctica requiere como condición indispensable conectar el saber disciplinario con estrategias específicas de enseñanza y evaluación de dicho conocimiento (Vergara Díaz y Cofré Mardones, 2014). Esto potenciaría la capacidad de los profesores en formación para diseñar unidades de aprendizaje pertinentes y debidamente contextualizadas.

Al parecer, el desarrollo de habilidades para reelaborar el saber disciplinario con fines pedagógicos tiene poca presencia en los planes educativos de los futuros profesores de Ll del nivel secundario, lo cual se verifica en la escasez de asignaturas didácticas específicas en las correspondientes mallas curriculares (Sotomayor y Gómez, 2017). Así, el impacto que dicha situación genera en las habilidades docentes de los futuros profesores es percibida por estos como una carencia relevante. En efecto, estudios realizados con estudiantes de Pedagogía Básica han constatado que poseen una actitud optimista frente a los contenidos en el área de L1 y de su capacidad para enseñarlos (Sotomayor et al., 2013), pero siempre desde un punto de vista del contenido. En el contexto español, Lorente Muñoz (2012) identifica una importante brecha entre lo enseñado a los profesores en la universidad y lo que ellos deben realizar en el aula escolar, caracterizando la formación en el área de lengua como de corte academicista. En el contexto latinoamericano, Romero y Concha (2017) constatan las dificultades que 
tienen los futuros docentes para darle una dimensión didáctica a los planteamientos teóricos. Lo anterior permite suponer que, en Chile, la formación lingüística en programas de formación inicial docente no dista mucho de esta aproximación teórica, desvinculada de una reelaboración didáctica necesaria para la futura labor de los profesores en formación.

Tal situación demanda un cambio en el quehacer de los académicos que enseñan contenidos disciplinares en carreras de Pedagogía. En la enseñanza de la lingüística para futuros profesores secundarios, el desafío formativo que estos deben asumir es la generación de situaciones de aprendizaje potenciadoras de su capacidad para operacionalizar, en términos didácticos, los contenidos lingüísticos, con el fin de ponerlos al servicio del análisis y la reflexión de ejemplares discursivos concretos para enriquecer las habilidades de comprensión y producción de textos (Mendoza Fillola, 1998). En otras palabras, los saberes disciplinares en el área de la lengua debieran articularse con un enfoque didáctico, pues, según Geegerat Vera y Vásquez Palma (2008), esta sería la única manera de enseñar a enseñar. Dicho planteamiento es coherente con lo que plantean los estándares de la formación inicial propuestos por el Ministerio de Educación de Chile, Mineduc, que recalcan la dimensión didáctica como un aspecto central en una formación inicial docente de calidad (Sotomayor y Gómez, 2017).

Así pues, configurar una didáctica de la lingüística para profesores en formación es una tarea ineludible. Sin embargo, hay una carencia de estudios en esta línea (Moya Muñoz, 2019). Existen algunas propuestas centradas en la enseñanza de la gramática (Meneses et al., 2017) y algunas investigaciones vinculadas con las concepciones de los académicos respecto de la escritura (Correa et al., 2013; Rubio, 2011) y el uso de textos multimodales en la formación inicial docente (Farías y Araya, 2015). Además, hay algunas obras como la de González Nieto (2001) orientadas a una lingüística para profesores, pero que constituyen una compilación de teorías sin ninguna reelaboración didáctica. De hecho, Sardi D'Arielli (2001) critica esta obra por abordar solo una necesaria "actualización teórica" (p. 121), pero que resulta insuficiente debido a la falta de consideración de "las situaciones particulares que se ponen en escena en la escuela” (p. 122). 
Para avanzar en la configuración de dicha didáctica de la lingüística para profesores en formación, es necesario indagar en torno a los significados construidos sobre su propio quehacer por parte de los académicos que enseñan esta disciplina. Dichos significados se relacionan con la puesta en discurso de sentidos, experiencias, creencias, valoraciones, actitudes, etc., es decir, aspectos subjetivos presentes, implícita o manifiestamente, en el modo de representar discursivamente la acción de enseñar lingüística a futuros profesores.

En consecuencia, el propósito de este artículo es describir los significados construidos por los académicos del área de lingüística de la Universidad de Santiago de Chile (Usach) respecto de la enseñanza de la disciplina a futuros profesores de castellano (en el nivel secundario). En esta investigación se adopta como supuesto que es necesario generar condiciones de diálogo para reflexionar acerca de las prácticas pedagógicas y así, a futuro, avanzar en la configuración de una didáctica de la lingüística en la formación de profesores de lengua materna. Para ello, se realizó una indagación de carácter cualitativo que posibilitó una puesta en discurso de la práctica pedagógica de dichos académicos, así como las vinculaciones que percibían en relación con la enseñanza de la L1 en el contexto escolar y el desarrollo de la competencia comunicativa de sus propios estudiantes.

\section{Marco teórico}

La investigación realizada asume que el modo en que los académicos conciben, reflexionan, valoran, diseñan y evalúan la enseñanza de la lingüística es un aspecto central. En efecto, la investigación especializada destaca la relevancia de la subjetividad configurada por los docentes en torno a los contenidos de enseñanza (Borg, 2003; Vaillant y Marcelo, 2015). Para que el académico del área de lingüística conciba su labor como la de un formador de formadores, debe identificar y problematizar la racionalidad en la cual asienta la enseñanza de la disciplina. En otras palabras, es necesario reflexionar en cuanto a la propia práctica docente para pasar desde lo que Freire (2012) denomina una educación bancaria, centrada en la transmisión del contenido, a una práctica problematizadora, centrada en el sujeto que aprende. Montenegro y Medina (2014) enfatizan la reflexión de los impactos que tiene en los futuros profesores el modelo de enseñanza utilizado y la organi- 
zación social de la clase, apuntando a la necesidad de superar la racionalidad técnica que subyace a la transmisión de contenidos (Groundy, 1998), la cual se asocia a la concepción de educación bancaria.

Coherente con esta perspectiva, usamos como marco de referencia, por un lado, la noción de consciencia lingüística (CL) en el entendido que ayuda en el desarrollo de la competencia comunicativa de los sujetos. Por otro lado, consideramos los saberes lingüísticos implicados en la formación del profesorado de Ll, en la medida en que el conocimiento disciplinar es fundamental en la enseñanza universitaria.

\section{La consciencia lingüística y la consciencia lingüística del profesor}

La CL (denominada en otras tradiciones investigativas como reflexión metalingüística, reflexión sobre la lengua o consciencia metalingüística) apunta a la capacidad de un sujeto de centrar su atención en "cualquier aspecto relacionado con la lengua" (Cots et al. 2007, p. 20), es decir, se vincula con el desarrollo de la habilidad de observación de fenómenos lingüísticos sean estos de carácter formal o contextual y que, potencialmente, sirva de apoyo para el desarrollo de herramientas que faciliten el éxito en las actividades comunicativas que el sujeto lleva a cabo. Dicha observación se enriquece en la medida en que se desarrolle la capacidad metacognitiva del sujeto respecto de sus propias prácticas discursivas.

En el contexto de enseñanza de segundas lenguas, Svalberg (2007) identifica dos aspectos cognitivos relacionados con la CL, a saber, la atención y el darse cuenta (noticing en inglés). En esta línea, la atención sería una condición necesaria, pero no suficiente para el desarrollo de la CL, puesto que se requeriría un cambio en el comportamiento del sujeto, lo que permite señalar que se ha vuelto consciente. Dicho cambio se vería facilitado por el darse cuenta, es decir, un estado de alerta consciente centrado en el cambio del comportamiento lingüístico (Schmidt, 1994). Obviamente, las características lingüísticas implicadas en esta capacidad para darse cuenta dependerán de lo que se esté enseñando. Es importante destacar que los estudios se han centrado, en su mayoría, en el aprendizaje de las formas gramaticales dejando de lado otras características de la lengua (Andrews, 2007; Camps y Milian, 2000; Rodríguez, 2012; Svalberg, 2007) que ayudarían al desarrollo de la CL. 
La CL no sería una capacidad de términos absolutos, sino que se desplegaría en diferentes grados desde una actividad intuitiva con bajo nivel de consciencia hasta una actividad de reflexión del uso lingüístico a partir de un conocimiento explícito formal y técnico acerca de la lengua, es decir, metalingüístico (Culioli, 1990; Van Lier, 1995).

En el sistema escolar, se considera que la CL juega un rol relevante en el desarrollo de la competencia comunicativa por parte de los estudiantes secundarios (Andrews, 2007; Camps y Milian, 2000; Meneses et al., 2017; Rodríguez, 2012). Para desarrollarla, Cots et al. (2007) proponen la articulación de cuatro aspectos: énfasis en tareas comunicativas naturales, aprendizaje exploratorio, aprendizaje de conceptos lingüísticos relevantes y autonomía en el aprendizaje.

En concreto, los profesores de L1 deben partir reconociendo los esquemas de entrada (implícitos) que poseen sus estudiantes - es decir, su repertorio lingüístico- con el fin de ampliarlos. Para lograrlo, es fundamental que posean un conocimiento lingüístico explícito (Meneses et al., 2017). En otras palabras, tal como como señalan Meneses et al. (2017) los profesores requieren de un conocimiento especializado y práctico para mejorar la competencia comunicativa de los estudiantes, gracias al desarrollo de la CL (Andrews, 2007; Camps y Milian, 2000; Rodríguez, 2012; Van Lier, 1995).

En esta línea, la consciencia lingüística del profesor (CLP) es un concepto esencial para comprender la naturaleza de la formación de un profesor de L1 (Andrews, 2007; Borg, 2003). La CLP se refiere a la interfaz entre lo que los profesores saben o necesitan saber respecto del lenguaje y su práctica pedagógica (Andrews, 2007). La CLP se crea a partir de la combinación de tres esferas (Andrews, 2007; Lindahl, 2019), a saber:

- usuario, relacionada con el dominio del idioma por parte del profesor y el conocimiento en cuanto a las variedades de la lengua usadas por los estudiantes;

- analista, vinculada con la comprensión que tiene el profesor acerca de la lengua en tanto sistema; y

- pedagogo, concebida como la capacidad del profesor para planificar los contenidos de enseñanza. 
A juicio de los expertos, la CLP es un término que ha estado en constante evolución (Andrews, 2006). En un principio, se refería solamente a los conocimientos en cuanto a la lengua que debía tener un profesor, mientras que en la actualidad se incorporan las cogniciones específicas que poseen los docentes, entre las que se cuentan la lengua en general, la lengua que enseña y el grado de desarrollo de la lengua de los estudiantes con los que trabaja. El trabajo desarrollado bajo el alero de la primera de las aproximaciones es denominado en la literatura como foco en la forma (Andrews, 2007; Cots et al., 2007). Se basa en el principio de que el rol del conocimiento sobre el lenguaje es que los estudiantes centren su atención en las propiedades específicas de la lengua, lo que tendría un efecto positivo tanto en el grado de adquisición de la lengua como en su nivel de competencia. Esto va de la mano con las prácticas de muchos profesores de lengua, quienes parecen basarse en un modelo de enseñanza en el que el conocimiento explícito es un facilitador del conocimiento implícito, ayudando a los alumnos, en primer lugar, a darse cuenta de ciertas propiedades formales de los fenómenos lingüísticos que no perciben fácilmente (Cots, 2017).

Desde un punto de vista procedimental, la CLP se compone de tres tipos de factores: actitudinales, contextuales y profesionales (Andrews, 2007). Los actitudinales son aquellos relacionados con la confianza y preparación para prestar atención a los problemas de la lengua (Sangster et al., 2013). En segundo lugar, los contextuales se refieren a la manera en que los profesores perciben el impacto de las constricciones impuestas por las condiciones de trabajo (programa de asignatura, tiempo para impartir clases, etc.). En tercer lugar, los factores profesionales se componen de las cogniciones en cuanto a la materia, competencia lingüística, consciencia del lenguaje desde la perspectiva de los estudiantes y experiencia docente. Ahora bien, en relación con las cogniciones (que es el foco de esta investigación), se puede sostener que estas aluden a lo que se ha denominado el "lado escondido" de la enseñanza o "vida mental" de los profesores y en la manera en que estas moldean un proceso de enseñanza y aprendizaje efectivo (Andrews, 2007; Borg, 2003). Las cogniciones respecto de la materia son consideradas el corazón del modelo de CLP (Andrews, 2007). Estas están interrelacionadas con otras para influir en el tratamiento de la gramática por parte del profesor. El conocimiento acerca del contenido (o su ausencia) juega un rol central en el pensamiento 
de los profesores y su proceso de toma de decisiones. Además, este conocimiento está ligado con una serie de cogniciones relacionadas: sentimientos personales de la gramática y su pedagogía; percepciones de los sentimientos de los estudiantes en cuanto a la gramática; comprensión del rol de la gramática en la comunicación y su significado en la adquisición de la lengua, consciencia de las opciones para tratar la gramática en instrucciones formales; comprensión de las expectativas del entorno (padres, escuela, estudiantes, etc.) en relación con la gramática y su pedagogía.

\section{Saberes lingüísticos implicados en la formación del profesorado de L1}

Si bien se sabe que los saberes de los formadores de docentes son de diversa naturaleza (Tardif, 2010; Vezub, 2016), existe consenso en que el conocimiento disciplinar es importante al momento de la enseñanza en la universidad, en tanto se persigue que los futuros profesores no sean meros aplicadores de una teoría específica en su futura práctica, sino que construyan su identidad a partir de su capacidad de reflexión que derivan en saberes específicos generados a partir del desempeño profesional (Tardif, 2010). Dicha posición instaura una doble necesidad: identificar, por un lado, las prácticas de enseñanza disciplinar que se realizan en los contextos universitarios (Montenegro y Medina, 2014); y, por otro, reconocer las áreas del conocimiento necesarias para la formación docente. Este último punto no ha estado lo suficientemente claro en las estructuras curriculares de las carreras de Pedagogía en Castellano. Tal como han planteado Sotomayor y Gómez (2017), más de la mitad de las asignaturas de las mallas de estudio de las carreras de Pedagogía en Castellano están constituidas por asignaturas disciplinares (lingüística y literatura), no obstante, se desconoce en qué proporción se encuentran representadas y cuáles son las áreas específicas que están involucradas. Concretamente en el área de lingüística, no se sabe cuáles son las áreas que deben ser consideradas en la formación de profesores.

Para diversos autores (Bronckart, 1985; Dolz, 2011; Lomas et al., 1993; Meneses et al., 2017) la definición de los saberes lingüísticos indispensables para la formación inicial docente de L1 debe partir por comprender cuál es la función de un futuro profesor en el aula. En la 
actualidad, luego de haber transitado durante mucho tiempo a través de una enseñanza basada en la memorización de reglas gramaticales que se aplicaban a la resolución de determinadas tareas no naturales de comunicación (Bronckart, 1985), existe acuerdo cuando se precisa que una de las principales labores del profesor es la de apoyar el desarrollo de la competencia comunicativa del alumnado, considerándola como "el conjunto de procesos y conocimientos de diverso tipo -lingüísticos, sociolingüísticos, estratégicos y discursivos-que el hablante/oyente/escritor/lector deberá poner en juego para producir o comprender discursos adecuados a la situación y al contexto" (Lomast al., 1993, p. 15).

A partir de esta consideración, que además es la defendida por el currículo nacional, la literatura especifica determinados saberes disciplinares, más allá del aspecto gramatical que se ha detallado en la sección anterior y que apoya el desarrollo de la CL (Rodríguez, 2012). En primer lugar, Bronckart (1985) parte de la noción de que la lingüística aplicada no es capaz de resolver las problemáticas propias de la enseñanza de la Ll, puesto que en esta ha prevalecido una perspectiva aplicacionista de la enseñanza, en otras palabras, tal como señala Tardif (2010): “[El modelo aplicacionista] está ideado según una lógica disciplinaria y no según una lógica profesional centrada en el estudio de las tareas y realidades del trabajo de los docentes" (p. 200). Por lo tanto, para Bronckart (1985) es la didáctica de la lengua la que debe hacerse cargo, como disciplina, de la enseñanza de la lengua. Para lograrlo, a su juicio, los docentes deben ser formados en tres áreas indispensables de la lingüística: teorías del lenguaje, puesto que entrega a los profesores un metalenguaje que facilita la conceptualización de problemas relacionados con el uso lingüístico; técnicas que permitan el reconocimiento de las unidades lingüísticas de una lengua; y la enseñanza de la lengua desde la variación.

En segundo lugar y en una perspectiva similar, Tusón (1993) identifica cuatro saberes: la descripción del sistema lingüístico; los factores que condicionan la adquisición de la lengua; los factores sociales que articulan los usos lingüísticos y los métodos de enseñanza específicos. En tercer lugar, Schleppegrall (2020) parte de la idea de que los estudiantes llegan a la sala de clases con un repertorio lingüístico ya desarrollado, al que se le pueden ir agregando variedades de len- 
gua diferentes. Para lograrlo, el profesor debería estar formado en el reconocimiento de la variación sobre los textos y tareas disciplinarias (porque el profesor de lengua debe estar en el centro de la adquisición del conocimiento de cualquier área) y en el uso de un metalenguaje significativo que le ayude a sus estudiantes a conseguir sus propósitos comunicativos.

Para finalizar esta revisión de cuatro autores respecto de los saberes implicados en la formación docente, es necesario detallar los componentes gramaticales indispensables en dicha formación que ayuden en el desarrollo de la competencia comunicativa de los estudiantes. Van Rijt y Coppen (2017) plantean dividir estos saberes en tres grupos: estructurales (relacionados con las funciones, estructuras y categorías sintácticas), semánticos (relacionados con la coherencia) y relacionales (cuyo rol es vincular elementos lingüísticos, como estructura informacional, gramaticalización y orden de las palabras).

En resumen, dos son las direcciones fundamentales en la formación inicial de profesores de lengua materna: el concepto de variación, entendido en un sentido amplio, y el conocimiento del sistema lingüístico que facilite la reflexión en cuanto a la propia lengua y su posibilidades de significación.

\section{Metodología}

La investigación corresponde a un estudio de caso cualitativo (Simons, 2011; Stake, 1998), pues lo que se buscó era comprender los sentidos que los académicos del área de lingüística de la Usach le asignaban a la acción de enseñar dicha disciplina en un contexto específico: la formación profesional de futuros profesores de Castellano. Cabe aclarar que no se observó la práctica de enseñanza, sino el discurso elaborado acerca de tal práctica, es decir, se generaron condiciones para que ellos verbalizaran sus experiencias pedagógicas sin intentar imponer un punto de vista particular, pues se entiende que en el relato de lo vivido se van explicitando significados e interpretaciones, tanto manifiestos como subyacentes (Creswell, 2007; Kornblit, 2007).

Los sujetos entrevistados fueron 11 académicos que imparten asignaturas del área de lingüística y desarrollo de habilidades de comprensión y producción de textos en la carrera de Pedagogía en Castellano. La Tabla 1 caracteriza a los entrevistados. 
Tabla 1

Descripción de los sujetos

\begin{tabular}{lllll}
\hline & $\begin{array}{c}\text { Rango } \\
\text { etario }\end{array}$ & Género & $\begin{array}{c}\text { Formación } \\
\text { profesional }\end{array}$ & $\begin{array}{c}\text { Máximo grado } \\
\text { obtenido }\end{array}$ \\
\hline Académico 1 & $50-60$ & Masculino & Profesor & Doctorado \\
\hline Académico 2 & $40-50$ & Femenino & Profesor & Magíster \\
\hline Académico 3 & $30-40$ & Femenino & Profesor & Doctorado \\
\hline Académico 4 & $30-40$ & Masculino & Profesor & Magíster \\
\hline Académico 5 & $50-60$ & Femenino & Profesor & Licenciado \\
\hline Académico 6 & $60-70$ & Masculino & Profesor & Magíster \\
\hline Académico 7 & $50-60$ & Masculino & Profesor & Magíster \\
\hline Académico 8 & $40-50$ & Masculino & Profesor & Doctorado \\
\hline Académico 9 & $40-50$ & Femenino & Licenciado & Doctorado \\
\hline Académico 10 & $30-40$ & Femenino & Profesor & Magíster \\
\hline Académico 11 & $40-50$ & Femenino & Profesor & Magíster \\
\hline
\end{tabular}

Fuente: Elaboración propia.

El instrumento empleado fue una entrevista semiestructurada que tenía cuatro temas de conversación: la función del profesor de castellano en el sistema escolar, la enseñanza de la lingüística para profesores de castellano, el desarrollo de la CL en la formación inicial docente y la evaluación del desempeño del alumnado de la carrera. En cada uno de ellos, se evitó inducir la respuesta de los entrevistados, no empleando valoraciones en las preguntas gatillantes y otorgándoles el tiempo y la libertad para que pudieran explayarse si lo deseaban. En esta línea, se adoptó el criterio de neutralidad empática (Patton, 2015), el cual supone generar un clima de confianza que estimula la verbalización de los entrevistados y controlando las posibles valoraciones de dichas verbalizaciones por parte del entrevistador.

Las entrevistas fueron transcritas para el análisis de su contenido, el cual se realizó mediante un proceso inductivo, es decir, la construcción de categorías y de una descripción guiada por el propio discurso de los entrevistados. En los segmentos discursivos elegidos, se destacó en negrita aquellas ideas centrales que pudieran ser relevantes para comprender los significados expresados por los entrevistados.

La lectura y relectura de las entrevistas permitió la identificación de dos polos opuestos en torno al lugar desde el cual el entrevistado 
desplegaba su discurso: a) un polo centrado en la disciplina en el que el entrevistado evidencia un fuerte compromiso con ella y un escaso conocimiento del sistema escolar y b) un polo centrado en la interacción disciplina-didáctica donde el entrevistado evidencia un fuerte compromiso con la disciplina y un amplio conocimiento del sistema escolar y la función que en él desarrolla el profesor de Castellano.

Posteriormente, se seleccionaron los académicos que prototípicamente representaban los polos descritos anteriormente y se hizo un análisis exhaustivo de su discurso orientado a la construcción de categorías (Kornblit, 2007), las que emergieron fueron: enseñanza de la lingüística, visión acerca de la lingüística, cobertura disciplinar en la malla de la carrera, condiciones de operación en la carrera, rol del profesor de Castellano, caracterización de los estudiantes de la carrera, caracterización de los académicos de la carrera. Considerando dichas categorías, se efectuó una síntesis del punto de vista de cada uno de estos académicos, concretado en un organizador gráfico que pudiera explicitar la racionalidad presente en el discurso de estos entrevistados.

Finalmente, se utilizaron las categorías indicadas en el párrafo anterior para analizar las entrevistas de los académicos restantes e hipotetizar su grado de cercanía a uno de los dos polos descritos.

Es importante indicar que dicha categorización y descripción fue realizada por los dos investigadores de este estudio en conjunto, quienes discutieron cuando había algunas diferencias con el fin de llegar a un consenso, el cual era refrendado por la apelación al propio discurso de los entrevistados, evitando así sobreinterpretar o tergiversar sus puntos de vista.

\section{Resultados}

\section{Dos polos en la construcción del relato de los académicos}

En términos generales, el relato de los entrevistados se sitúa en dos polos que, prototípicamente, fue evidenciado en el discurso de dos académicos con visiones contrarias respecto de la enseñanza de la lingüística para futuros profesores. Estos polos son: a) la enseñanza de la lingüística en tanto saber científico válido en sí mismo y b) la enseñanza de la lingüística centrada en la formación de un sujeto activo 
consciente de sus prácticas discursivas. A continuación, se describirá cada uno de ellos.

\section{La enseñanza de la lingüistica en tanto saber científico válido en símismo}

El discurso de este académico se articula sobre la disciplina lingüística sin referencia a la futura labor docente de los profesores en formación, la cual prácticamente se desconoce. De hecho, se considera que el profesor de enseñanza media debiera enseñar la disciplina sin que medie una reelaboración didáctica de la misma. De este modo, se puede apuntar a la ampliación del repertorio lingüístico sin especificar concretamente cómo ello se podría lograr. Se concibe al estudiante de Pedagogía desde el déficit y que la labor de enseñanza con un alto grado de rigurosidad terminológica y la aplicación guiada de la teoría como ejercicio académico podría habilitarlo para definir, aplicar y orientar el uso lingüístico. En esta línea, lo central es el aseguramiento del desarrollo disciplinar mediante la concurrencia de académicos con un alto nivel de especialización y con la habilidad de aplicación. Esta visión se ha sintetizado en la Figura 1 y se desarrollará a continuación.

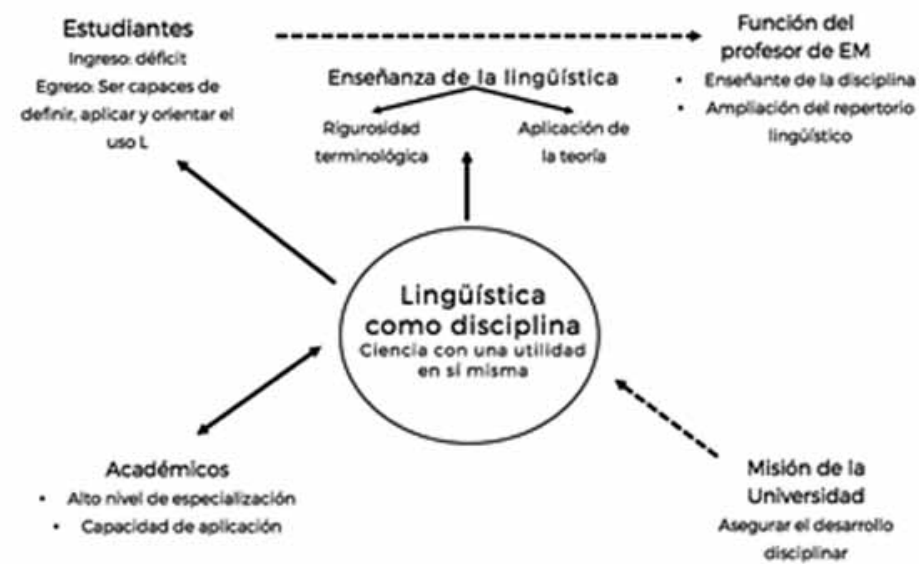

Figura 1. Articulación del discurso del académico que, prototípicamente, representa la enseñanza de la lingüística en tanto saber científico válido en sí mismo

Fuente: Elaboración propia.

El lugar de posicionamiento de este académico es de un alto compromiso con la disciplina y un bajo conocimiento del sistema escolar. 
En su discurso, pareciera que idealmente la formación docente debiera involucrar un conocimiento extenso y profundo del contenido lingüístico con el fin de formar un especialista que lo maneje tanto teórica como prácticamente.

Yo creo que toda, con excepción de algunas aplicaciones que tal vez sean muy... tecnológicas, como lingüística matemática, lingüística de corpus, que requiere... pero son como orientaciones posteriores. Yo creo que la lingüística que requiere es, en términos de escuelas, cualquiera que le dé una terminología precisa y rigurosa, o sea, no me molestaría si alguien formara, desde el punto de vista generativista, por ejemplo, y otro, una perspectiva estructuralista europea. A eso no, no le veo problema, con tal de que sea una terminología que pueda, que se pueda revisar, que se pueda observar y que se pueda aplicar (Académico 1).

Llama la atención que no haya en su discurso una especificación del contenido requerido por los profesores en formación ni una clara opción por un enfoque dado. Otro aspecto interesante es que quien decide el contenido a enseñar es el académico concebido como un especialista aislado, que no debate y logra acuerdo con otros en torno a los enfoques disciplinares a ser enseñados y requeridos por los futuros profesores. Dicho enfoque es conceptual con posibilidades de aplicación. En relación con los criterios de selección del contenido a enseñar, recalca que:

Yo creo que los criterios son dos: uno como lo que es básico en la disciplina ... Y otro, lo que tiene pertinencia para la formación de ellos... Con esos dos criterios yo me las arreglo, las cosas que dejo fuera, las dejo fuera porque o no son básicas de la disciplina o no tienen mucha aplicación y si es algo que no es muy básico de la disciplina, pero tiene aplicación, de alguna manera lo incorporo también (Académico 1).

El hilo conductor en la selección es la disciplina. En este sentido, el criterio "lo que tiene pertinencia para la formación de ellos" pareciera asociarse con la aplicación de la disciplina y no, necesariamente, con una reelaboración didáctica del saber. Subyace la idea de que lo que el profesor de L1 enseña es contenido disciplinar, por tanto, la aplicación de la teoría se constituye en un eje central de la docencia. Coherentemente, la malla curricular se evalúa en tanto la cobertura disciplinar tenga un espacio relevante: 
Yo creo que la formación está bien en general, está bien secuenciada. $\mathrm{Y}$ eso les permite ir aprendiendo de manera más o menos gradual y cuando ya son más grandes ya se ubican bastante bien, o sea, yo creo que tienen la formación que pueden recibir, es bastante buena, entonces y eso, claro, eso es una fortaleza para ellos (Académico 1).

En concreto, no se evidencia una visión crítica respecto del tipo de contenido requerido por un profesor en formación ni las posibilidades de articulación con lo pedagógico o didáctico concebido como un eje transversal, pues una buena formación docente se equipara a una buena formación disciplinar.

Desde esta perspectiva, el concepto de CL para este académico se vincula con el metalenguaje que se desarrolla gracias al conocimiento disciplinar y que se aplica al uso.

Yo creo que en todas las asignaturas, si uno los mira como por niveles del lenguaje, existe un uso y existe también consciencia acerca de que lo que estaba haciendo, lo que te da, en términos prácticos, el metalenguaje. Tú puedes tener reflexión acerca de los sonidos que estás utilizando... Tú usas la sintaxis, pero también puedes saber cómo la estás usando, si estás utilizando siempre una misma fórmula y que lo mismo se pueda hacer de otra fórmula, de otra forma, etc. $\mathrm{Y}$ a nivel discursivo también, cuando tú te preguntas cuál es el acto de habla que estoy realizando, por ejemplo... o sea, cuando tú tienes consciencia lingüística es porque utilizas la información lingüística para tu propia producción y para tu propia comprensión y eso yo lo considero fundamental, yo creo que el profesor debería ser un ejemplo... Esta consciencia lingüística yo creo que se desarrolla mediante la observación de la práctica lingüística a la luz de la teoría, de alguna teoría (Académico 1).

La CL se relaciona con la reflexión en cuanto al uso, pero dicha reflexión se guía teóricamente sin que se problematice el tipo de teoría que sea más pertinente. Subyacentemente, queda la idea de que la primacía corresponde al conocimiento teórico. En consecuencia, el perfil del académico ideal para la formación de futuros profesores es uno altamente especializado con capacidad de enseñar generando las posi- 
bilidades de aplicar lo aprendido. Este sería un nivel de especialización tan alto que, pareciera, observa los fenómenos fragmentariamente sin que haya integración de diferentes niveles de análisis.

Yo creo que los profesores deben ser cada vez más especialistas... o sea, el profesor que hace gramática, que hace fonética y que hace semántica, yo creo que se van en retirada. Ahora, concentrar a los profesores en una sola área o en dos relacionadas está bien... Especialización de los profesores, vinculación de los contenidos a la práctica profesional, yo creo que eso es algo que hay que avanzar, que algunos profesores lo hacen, no todos. Yo creo que todavía se sigue pensando, en algunos pueden tener en la cabeza todavía la idea de que es importante que aprenda bien esto ahora y que después lo va a aplicar, la idea es que lo aprenda bien aplicándolo (Académico 1).

Es interesante constatar que en el discurso de este académico no hay la posibilidad de generar situaciones de análisis que favorezcan una aproximación interdisciplinar al interior de la propia lingüística ni con otras disciplinas ligadas a lo literario, pedagógico y/o didáctico. Congruentemente, se concibe la labor del profesor de enseñanza media como un enseñante de la disciplina, pues enseñando lingüística es posible el desarrollo de la CL, fundamentalmente de habilidades de escritura y la rigurosidad conceptual.

Es importante destacar que la visión de este académico se configura desde una concepción particular de la lingüística como ciencia. En efecto, se opone ciencia y humanidades, en la cual la ciencia tendría un estatus epistemológico superior. En otras palabras, subyace un dejo de inferioridad de las humanidades y de la reflexión que ellas implican. Esto se podría asociar a la idea de una visión positivista de la lingüística, es decir, una ciencia libre de valores que estudia la lengua y su uso como si fueran cosas externas independiente de los sujetos y sus contextos.

[En relación con una feria científica desarrollada por la Facultad de Ciencias] Y nosotros podríamos mostrar cosas espectaculares ahí, en esa misma feria, como científicos que somos, pero claro, nos sentimos como en otro mundo, más de las humanidades, estamos reflexionando, mirando pa'l techo (Académico 1). 


\section{La enseñanza de la lingüistica centrada en la formación de un} sujeto activo consciente de sus prácticas discursivas

El discurso de este académico se configura en torno al estudiante de Pedagogía en L1 concebido como un sujeto activo y consciente de sus prácticas lingüísticas. La enseñanza de la lingüística se representa como una acción reflexiva y rigurosa que se sustenta en la interacción con dicho sujeto. En otras palabras, la enseñanza de la lingüística empodera a los estudiantes para que reflexionen acerca de sus prácticas discursivas y, al mismo tiempo, considera dichas prácticas como situaciones de aprendizaje, apuntando a familiarizarlos con lo que él estima como conceptos basales de la disciplina desde el punto de vista teórico y práctico y a develar cómo dichos conceptos se relacionan con los saberes propuestos por el marco curricular. En este sentido, existe un conocimiento de los fines educativos del marco y del enfoque de la asignatura de lenguaje, y se tiene como referencia el rol que el profesor de lenguaje tiene en el sistema escolar. Este modo de concebir la enseñanza de la Lingüística se asienta en una visión antropológica del lenguaje, es decir, no se le considera un mero instrumento de comunicación, sino que una facultad inherentemente humana que constituye a los sujetos, su interacción y el espacio socialmente compartido. Congruentemente, la lingüística constituye un saber sobre la lengua que es histórico, es decir, una aproximación sometida a la dinámica de continuidad y cambio. Esta visión se ha sintetizado en la Figura 2 y se desarrollará a continuación.

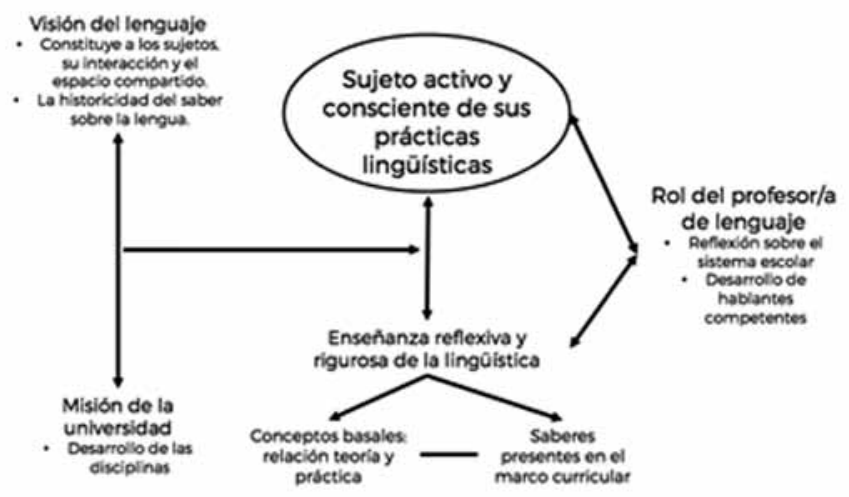

Figura 2. Representación del discurso del académico que, prototípicamente, concibe la enseñanza de la lingüística centrada en la formación de un sujeto activo consciente de sus prácticas discursivas

Fuente: Elaboración propia. 
El lugar de posicionamiento de este académico es el diálogo entre la lingüística y la didáctica basado en un alto compromiso disciplinar y un alto grado de conocimiento del sistema escolar. Desde dicho posicionamiento, despliega una crítica a la enseñanza tradicional de la lingüística, pues esta transmite un saber fragmentario, centrado en lo conceptual y que genera un saber superficial en los futuros profesores. Para él, el profesor de lengua debe ser un especialista reflexivo y un didacta con un alto nivel de consciencia y sensibilidad al contexto, capaz de integrar saberes en el diseño de experiencias de aprendizaje.

Yo creo que un profesor de aula tiene dos obligaciones. Hay una obligación que tiene que ver con ampliar un repertorio disciplinar... pero, además, un profesor de lengua es un profe... es alguien que, junto con ampliar el repertorio o el capital, digamos... el gran universo de conceptos que pueden permitir que un chiquillo hable, lea o escriba, es alguien que tiene que ser capaz de apoyar a que ciertos comportamientos se modifiquen o cambien. ¿A qué me refiero con eso? Me refiero a que un profesor de lengua es alguien que tiene que estar súper atento a la actividad lingüística de los estudiantes y desde ahí asumir que, en realidad, por una parte, su función está en que los chiquillos sean capaces de observar productos de habla. Pero, además, integrar en qué medida, él en sí mismo es un generador de estos productos y, por lo tanto, mal haríamos en una clase de lengua si no estamos atentos a cómo es que los chiquillos están experimentando en sí mismos esos fenómenos. Si a un chiquillo no le pasa nada, luego de muchas clases y no ha incorporado una palabra nueva, no ha puesto en reflexión sus recursos del uso, de sus frases típicas, de sus comentarios... si luego de una clase de lenguaje un estudiante no se ha mirado a sí mismo respecto de él como productor de lenguaje, entonces, fracasamos como profes (Académico 3).

Al parecer la identidad de profesor está centrada en su rol transformador que posibilita la toma de consciencia de aspectos objetivos y subjetivos de la actividad lingüística. Dicho rol se sustenta en la generación de condiciones para que los estudiantes analicen y reflexionen acerca de la actividad discursiva propia y ajena orientada a impactar en los sentidos asignados a la acción. 
Entonces, cuando digo modifica conductas o comportamientos, no estoy hablando de veo que alguien está mal y lo hago hablar bien, no estoy pensando en eso, sino que estoy pensando en que, desde lo basal de la disciplina, yo amplío repertorio, amplío recursos, amplío conceptualizaciones, pero también, al mismo tiempo, apoyo la posibilidad de que el otro cambia su actuar a conveniencia de lo que necesite, a conveniencia de lo que requiera, ya sea para mirar cómo es su actividad lingüística o cómo es él como productor, digamos, de lenguaje, o ya sea para utilizarlo mejor en ciertos contextos, para usarlo distinto en ciertos contextos (Académico 3).

Un requisito fundamental para poder concretar en el aula esta visión es que los estudiantes de Pedagogía superen una visión fragmentada de la lingüística y puedan hacerla dialogar con otras disciplinas con el fin de visualizar diferentes producciones culturales como productos discursivos: "Es justamente la mirada desmembrada de las disciplinas la que atenta a que los objetivos de aprendizaje tengan lugar como deberían tener" (Académico 3).

Para este académico, la lengua tiene una función constitutiva tanto de los sujetos como de los espacios de interacción entre ellos, por lo cual su conocimiento es un soporte fundamental para el conocimiento de sí mismo, de los otros y abre la posibilidad al aprendizaje.

La consciencia lingüística justamente apela a entenderme a mí mismo como sujeto productor de lenguaje, hacedor de lengua y, además, entenderme como sujeto, como alguien que no solo convive con el lenguaje, sino a quien el lenguaje lo atraviesa. En la medida que yo soy consciente de eso, como parte constitutiva de mí, cierto, y que entiendo, digamos, cómo es que, de alguna manera, el lenguaje me constituye, me forma y en que medida ese lenguaje al ponerse en relación con otros convive con tras constituciones de lenguaje de los otros sujetos (Académico 3).

La concepción de este académico apunta a que el lenguaje es un espacio de convivencia en el que se configuran y despliegan los sujetos en interacción, en el cual conforman su propia subjetividad y la expresan. En otras palabras, no se concibe al individuo como un ente aislado, sino como un ente que en sí es social. El uso de la lengua, por tanto, está condicionado socialmente no como un instrumento externo, sino 
como una facultad desarrollada en la vivencia compartida e internalizada en la propia subjetividad.

En relación con la enseñanza de la lingüística, este académico plantea cuatro preguntas que, según su parecer, son relevantes al momento de enseñar esta disciplina a futuros profesores.

Yo creo que la pregunta de qué es la lingüística está súper resuelta y no tengo ninguna duda e independiente de que los chiquillos sean más o menos avanzados en la asignatura... yo creo que los chiquillos pueden atender a esa pregunta. ¿Qué significa saber lingüística? Yo creo que ya es una pregunta que empieza a tener como... cierto flaqueo. Ahora, isi es necesario saber lingüística? Claro la articulación que van a hacer los chiquillos va a estar asociada respecto si está demandado en el referente curricular, si un chiquillo dice que no es necesario saber lingüística habiendo leído los objetivos de aprendizaje, está muerto. Yo creo que no, yo creo que sí creen, que sí entienden que es necesario saber lingüística. Ahora, ¿qué de la lingüística es importante que aprenda un chiquillo? No sé si está tan reflexionado (Académico 3).

De las preguntas planteadas, parecieran ser dos las que, desde el punto de vista de los académicos que enseñan la disciplina, no han sido suficientemente reflexionadas: ¿qué significa saber lingüística?, ¿qué de la lingüística es importante que aprenda un futuro profesor de L1? Respecto de la primera pregunta, este académico articula una importante crítica: "Yo siento que hay un carácter asociado a la formación que sigue estando como montado en una perspectiva bien tradicional, bien memorístico, bien como... alojado en los conceptos" (Académico 3).

El enfoque conceptual en la enseñanza de la lingüística no favorece necesariamente un saber lingüístico que pueda movilizarse en la línea del enfoque problematizador anteriormente descrito, constituiría más bien una suerte de conocimiento inerte que no permite el diálogo con las metas de aprendizaje.

Yo creo que los chiquillos pueden apelar o pueden aludir a conceptos de la lingüística. Ahora, tengo dudas de si son capaces cuando leen una meta de aprendizaje, un objetivo de aprendizaje, si logran identificar cuáles son los conceptos de la lingüística que están detrás (Académico 3). 
Según este académico, la enseñanza de la lingüística tiene que ser coherente con el perfil de egreso, los estudiantes van a ser profesores de lengua y ese debiera ser un consenso mínimo sobre el cual los académicos seleccionen, secuencien y enseñen conceptos y procedimientos basales de la disciplina. Dicho consenso no se ha trabajado explícitamente.

En relación con la lingüística a enseñar, este académico opta por un enfoque discursivo que, holísticamente, movilice recursos analíticos de diversos niveles para abordar fenómenos concretos en la perspectiva de enriquecer los recursos de los usuarios, develar sentidos y ampliar la CL.

Yo creo que la lingüística de un futuro profe de la Usach tiene que ser justamente una lingüística que atienda como a los actos o a los productos... como respetando su naturaleza y eso obliga a que sea una lingüística que está en diálogo con todas sus intersecciones... tiene que ser una lingüística que de alguna manera permita... tanto descomponer como componer... y tiene que ser, también, una lingüística que, de alguna manera, vaya ofreciendo sentido desde los recursos para un chiquillo como actor o como hablante... Entonces tiene que ser una lingüística que de alguna manera ponga en permanente diálogo la abstracción con la concreción. No me lo imagino de otra manera (Académico 3).

En esta línea, este académico concibe la lingüística como una disciplina humanista al servicio del potencial de ser humano para comprenderse a sí mismo y al mundo que habita. Desde esta perspectiva, es necesario concebir la lengua y su uso en interacción con un proyecto en que el ser humano se realiza en comunidad con otros, es decir, intersubjetivamente toma consciencia de sí mismo y de sus posibilidades de ser en el mundo.

Cualquier acto de habla es como el resultado de tu presencia en el mundo. Entonces, para mi gusto como tener consciencia respecto de en qué medida esa presencia en el mundo tiene una huella que es comunicativa, tiene una huella que es de lenguaje, ¿cierto?, y no es ajena, no está en los libros... no está en los libros, no está en las clases, sino que es parte de mi cotidiano. Entonces, claro si tú me preguntaí [sic] desde el desafío de la lingüística, el gran desafío de la lingüística es que un chiquillo sea capaz de entender que todos 
los fenómenos que tienen lugar... son parte de mi día a día. Están en mí, están en mí... en mi práctica de manera como indiscutible, irrenunciable desde que despierto hasta que me acuesto. $\mathrm{O}$ sea, incluso si lo pensáramos en el ámbito de los sueños... todo puede constituir un acto lingüístico (Académico 3).

\section{La distribución de los académicos en los dos polos descritos}

De los dos polos descritos (ver Figuras 1 y 2), el segundo, es decir, aquel que centra la enseñanza de la lingüística en relación con las prácticas discursiva de los sujetos, es la tendencia que domina en el grupo de entrevistados. En efecto, seis de ellos tienden a acercarse a esta postura, mientras que tres de ellos claramente se ubican en la postura opuesta. Cabe indicar que hay dos académicos cuyo discurso tiene elementos de ambas posturas, sin acercarse claramente a ninguna de ellas.

Congruentemente, la mayor parte de los académicos tiende a considerar la lingüística como una base de referencia para comprender fenómenos discursivos, por lo cual postulan una reflexión para una transferencia con sentido a los estudiantes concebidos como usuarios de la lengua y dentro de un enfoque comunicativo. Por ello, consideran que los contenidos de lingüística en la formación inicial docente deben apuntar a la reflexión y estar en relación con el currículo escolar. Por el contrario, los académicos que se ubican en el primer polo consideran que se debe enseñar lingüística como disciplina en el sistema escolar como un bagaje mínimo, útil en sí mismo, considerando aspectos teóricos y prácticos.

En general, todos los académicos coinciden en que la responsabilidad por la aplicación de los contenidos lingüísticos reside en ellos mismos y que dichos contenidos son de alta complejidad. En términos metodológicos, un grupo de sujetos (académicos 1, 2, 4, 9 y 10) postula un enfoque conceptual centrado en el conocimiento declarativo y procedimental versus otro grupo (académicos 3, 5, 6, 7, 8 y 11) que plantea un enfoque de análisis textual dirigido a generar condiciones para la reflexión en torno a diversos usos lingüísticos.

Los académicos tienden a coincidir en que el propósito de enseñanza es facilitar una síntesis general de la disciplina, con un marcado 
énfasis funcional que relacione sistema y uso. Para ellos, el objetivo es proporcionar un panorama riguroso de los diferentes niveles de análisis lingüísticos y de las interdisciplinas. Cabe indicar que una mayor parte (8 de los 11 académicos) enfatiza la necesidad de una mejor coordinación de la puesta en práctica de la malla curricular que permita evitar repeticiones innecesarias y superar el enfoque meramente conceptual, puesto que se tiende a calificar la formación de los futuros profesores como meramente teórica y abstracta.

En relación con los criterios de selección de los contenidos lingüísticos en cada una de las asignaturas, existe consenso entre los académicos en torno a la incorporación de los conceptos básicos fundamentales. Otros criterios indicados por algunos académicos se refieren al conocimiento útil para el análisis y la transferencia didáctica.

La mayor parte de los académicos (6 de ellos) tienden a calificar la lingüística como una disciplina científica que proporciona un conocimiento necesario sobre la lengua y permite un uso reflexivo de ella. En esta línea, se tiende a definir la CL como un conocimiento explícito de carácter metalingüístico que favorece un manejo estratégico de la lengua, orientado a superar el sentido común y favorecer la comprensión de fenómenos discursivos y su debida adecuación al contexto. Dicha CL se desarrollaría mediante la observación, análisis y reflexión de la práctica discursiva concreta propia y ajena. Contrariamente, solo dos sujetos (académicos 1 y 2 ) postulan que se desarrolla mediante la aplicación de teorías. Es importante indicar que para todos los académicos entrevistados la CL supone una sensibilidad tanto a la norma como al contexto.

Respecto de la labor del profesor de Ll, los académicos coinciden en que este debiera tener una formación lingüística de buen nivel y que - según manifestó la mayor parte de ellos-, les permita contar con un bagaje que posibilite la reflexión en torno al uso lingüístico en el espacio escolar para ampliar los recursos comunicativos del alumnado, potenciando sus capacidades de comprensión y producción.

\section{Conclusiones}

De acuerdo con el discurso de los académicos, se constata la carencia, en el caso estudiado, de instancias de coordinación y debate enfocadas en los modos en que se enseña la lingüística en el contexto de la 
formación de profesores de Ll. Esto reduce las posibilidades de innovación y colaboración recíproca que permitan reelaborar el contenido lingüístico en función del rol del profesor en el sistema escolar. De hecho, pareciera ser que no se abordan fenómenos discursivos desde los distintos niveles de análisis y desde las interdisciplinas con el fin de generar una visión comprensiva y rigurosa de dichos fenómenos. Esto significa que se les asigna a los futuros profesores la responsabilidad en la construcción de una visión integrada de la disciplina que permita un abordaje holístico de fenómenos discursivos.

Se evidencia una tensión no abordada entre una visión aplicacionista de la disciplina de raigambre positivista versus una visión de reelaboración didáctica del contenido basada en un enfoque humanista. Es interesante hacer notar que el enfoque aplicacionista ha sido cuestionado, puesto que se articula sobre una lógica disciplinaria que desconoce la futura labor del profesor (Bronckart, 1985; Tardif, 2010). En este sentido, es necesario articular la formación lingüística a partir de una racionalidad centrada en el desarrollo de competencias profesionales. A pesar de que la mayor parte de los académicos entrevistados critica el enfoque conceptual de la enseñanza de la lingüística por ser este abstracto y teórico, pareciera ser que en la práctica no lo han superado, por lo cual cabe preguntarse si es que esto obedece a una reproducción de la forma en que aprendieron la disciplina y que impide eventuales innovaciones en el modo de enseñanza.

Respecto de la CL, los entrevistados expresan una noción con distintos grados de profundidad, no obstante, pareciera ser que no es una noción basal que impacte en sus prácticas pedagógicas de enseñanza de la disciplina. En otras palabras, la CL no ha significado un posicionamiento desde el cual dichos académicos establecen los criterios de selección de contenidos lingüísticos, la secuencia de actividades de aprendizaje y los indicadores de evaluación utilizados.

En la línea de dar continuidad a los insumos necesarios para una configuración de una didáctica de la lingüística en la formación de profesores de L1, se hace necesario contrastar el discurso de los académicos con sus prácticas de aula y, además, enriquecer los resultados con el punto de vista de otros actores relevantes en el proceso, tales como estudiantes y egresados. Paralelamente, es preciso indagar acerca de prácticas alternativas de la enseñanza de la lingüística para pro- 
fesores, con el fin de identificar experiencias que pudieran ser útiles al momento de configurar propuestas didácticas orientadas a profundizar el saber lingüístico de los futuros profesores.

\section{Referencias bibliográficas}

Agencia de la Calidad de la Educación. (2016). Estudios sobre formación inicial docente (FID) en Chile. https://bibliotecadigital. mineduc.cl/bitstream/handle/20.500.12365/2131/mono955.pdf? sequence $=1$ \&isAllowed $=\mathrm{y}$

Andrews, S. (2006). The evolution of teachers' language awareness. Language Awareness, 15(1), 1-19. https://doi. org/10.1080/09658410608668846

Andrews, S. (2007). Teacher language awareness. Cambridge University Press.

Bahamondes, G., Merino, C., y Espinoza, A. (2021). Didáctica de la lengua y la literatura en la formación de profesores en Chile, Tejuelo, (33), 217-248. https://doi.org/10.17398/19888430.33.217

Borg, S. (2003). Teacher cognition in language teaching: A review of research on what language teachers think, know, believe, and do. Language Teaching, 36(2), 81-109. https://doi. org/10.1017/s0261444803001903

Bronckart, J. -P. (1985). Las ciencias del lenguaje: ¿un desafío para la enseñanza? Unesco. https://archive-ouverte.unige.ch/unige:86384/ATTACHMENT01

Camps, A. y Milian, M. (2000). La actividad metalingüística en el aprendizaje de la escritura. En M. Milian y A. Camps (comps.), $E l$ papel de la actividad metalingüistica en el aprendizaje de la escritura (pp. 7-37). Homo Sapiens.

Correa, R., Tapia, M., Neira, A., y Ortiz, M. (2013). Teorías personales de docentes de lengua sobre la enseñanza de la escritura en sistema de educación pública chilena. Trabalhos em Linguística Aplicada, 52(1), 165-184. https://doi.org/10.1590/ s0103-18132013000100010

Cots, J. (2017). Knowledge about language in the mother tongue and foreign language curricula. En J. Cenoz, D. Gorter, y S. May (Eds.), Language Awareness and Multilinguism (pp. 31-42). Springer. 
Cots, J., Armengol, L., Arnó, E., Irún, M., y Llurda, E. (2007). La conciencia lingüistica en la enseñanza de lenguas. Graó.

Creswell, J. W. (2007). Qualitative inquiry and research design. SAGE.

Culioli, A. (1990). Pour une linguistique de l'énonciation. Opérations et représentations. Ophrys.

Dolz, J. (2011). Describir la actividad docente: un punto de vista didáctico para comprender el trabajo del profesor en el aula. En J. Vallès Villanueva, D. Álvarez Rodríguez y R. Rickenmann del Castillo (Eds.), L'activitat docent. Intervenció, innovació, investigació (pp. 97-114). Documenta Universitaria.

Farías, M. y Araya, C. (2015). Hacia una caracterización de los textos multimodales usados en formación inicial docente en lenguaje y comunicación. Literatura y lingüistica, (32), 283-304. https://doi.org/10.4067/s0716-58112015000200015

Freire, P. (2012). Pedagogía del oprimido. Siglo XXI.

Gaete, A., Gómez, V., y Bascopé, M. (2016). ¿Qué le piden los profesores a la formación inicial docente en Chile? Temas de la agenda pública, (86), 1-18.

Geeregat Vera, O. y Vásquez Palma, O. (2008). Crisis y temporalidad en la formación inicial de profesores de lenguaje y comunicación. Estudios pedagógicos (Valdivia), 34(2), 87-98. https:// doi.org/10.4067/s0718-07052008000200005

González Nieto, L. (2001). Teoría lingüística y enseñanza de la lengua. Cátedra.

Groundy, S. (1998). Producto o praxis del currículo. Morata

Kornblit, A. L. (2007). Metodologías cualitativas en ciencias sociales. Modelos y procedimientos de análisis. Biblos.

Lindahl, K. (2019). Teacher language awareness development and its implications for new educators. The New Educator, 15(2), 85100. https://doi.org/10.1080/1547688x.2018.1526356

Lomas, C., Osoro, A. y Tusón, A. (1993). Las ciencias del lenguaje, competencia comunicativa y enseñanza de la lengua. Paidós.

Lorente Muñoz, P. (2012). Análisis crítico de la formación del profesorado de lengua castellana y literatura para la docencia en el siglo XXI. Didáctica, innovación y multimedia, (23), 1-15. https://www.raco.cat/index.php/DIM/article/view/258166

Mendoza Fillola, A. (1998). Marco para una didáctica de la lengua y la literatura en la formación de profesores. Didáctica (Lengua y 
literatura), (10), 233-270. https://dialnet.unirioja.es/servlet/ articulo? codigo $=148726 \&$ orden $=1$ \&info $=$ link

Meneses, A., Hugo, E., Acevedo, D., y Ávila, N. (2017). Gramática para profesores. Consideraciones metalingüisticas para el aprendizaje. Ediciones de la Pontificia Universidad Católica de Chile, PUC.

Montenegro, H. y Medina, L. (2014). Polifonía discursiva y procesos de cambio en los formadores de docentes: ¿Qué voces movilizan sus prácticas de enseñanza? Estudios pedagógicos (Valdivia), 40(número especial), 161-182. https://doi. org/10.4067/s0718-07052014000200010

Moya Muñoz, P. (2019). La lingüística en la educación superior: percepciones de los directores de la carera de Pedagogía en Lenguaje en Chile. Revista Espacios, 40(36), 1-16. http:// www.revistaespacios.com/a19v40n36/19403608.html

Patton, M. Q. (2015). Qualitative research y evaluation methods. Sage.

Rodríguez, C. (2012). La enseñanza de la gramática: las relaciones entre la reflexión y el uso lingüístico. Revista Iberoamericana de Educación, (59), 87-118. https://doi.org/10.35362/rie590458

Romero, S. y Concha, S. (2017). Formación docente en el área de lenguaje: experiencias en América Latina. Ediciones de la Universidad Diego Portales, UDP.

Rubio, M. (2011). Concepciones sobre la producción escrita en académicos que forman profesores básicos. Literatura y lingüistica, (23), 123-140. https://doi.org/10.4067/s071658112011000100008

Sangster, P., Anderson, C., y O'Hara, P. (2013). Perceived and actual levels of knowledge about language amongst primary and secondary student teachers: Do they know what they think they know? Language Awareness, 22(4), 293-319. https://doi. org/10.1080/09658416.2012.722643

Sardi D’Arielli, V. (2001). La lingüística aplicada: una mirada estrábica sobre la escuela. Lulú Coquette. Revista de Didáctica de la lengua y la literatura, 1(1), 121-126.

Schleppegrall, M. (2020). The language base for language teaching: What is the English to be taught as a content? Language Teaching Research, 24(1), 17-27. https://doi. org/10.1177/1362168818777519 
Schmidt, R. (1994). Deconstructing consciousness raising in search of useful definitions for applied linguistics. AILA review, (11), 11-26. http://nflrc.hawaii.edu/PDFs/SCHMIDT\%20Deconstructing\%20consciousness\%20in\%20search\%20of\%20useful\%20definitions.pdf

Simons, H. (2011). El estudio de caso: teoría y práctica. Morata.

Stake, R. (1998). Investigación con estudio de casos. Morata.

Sotomayor, C., Coloma, C. J., Parodi, G., Ibáñez, R., Cavada, P., y Gysling, J. (2013). Percepción de los estudiantes de pedagogía sobre su formación inicial. Magis. Revista Internacional de Investigación en Educación, 5(11), 375-32. https://www.redalyc.org/pdf/2810/281028437006.pdf

Sotomayor, C. y Gómez, G. (2017). La demanda de los estándares para egresados y el curriculum formativo en las carreras de pedagogía en lenguaje y comunicación en Chile. En S. Romero y S. Concha (Eds.), Formación docente en el área de lenguaje: Experiencias en América Latina (pp. 89-117). Ediciones de la Universidad Diego Portales, UDP.

Svalberg, A. M. (2007). Language awareness and language learning. Language Teaching, 40(4), 287-308. https://doi.org/10.1017/ s0261444807004491

Tusón, A. (1993). Aportaciones de la sociolingüística a la enseñanza de la lengua. En C. Lomas y A. Osoro (comps.), El enfoque comunicativo de la enseñanza de la lengua (pp. 55-68). Paidós.

Tardif, M. (2010). Los saberes del docente y su desarrollo profesional. Narcea.

Vaillant, D. y Marcelo, C. (2015). El ABC y D de la formación docente. Narcea.

Van Lier, L. (1995). Introducing language awareness. Penguin.

Van Rijt, J., y Coppen, P. A. (2017). Bridging the gap between linguistic theory and L1 grammar education-experts' views on essential linguistic concepts. Language awareness, 26(4), 360-380. https://doi.org/10.1080/09658416.2017.1410552

Vergara Díaz, C. y Cofré Mardones, H. (2014). Conocimiento pedagógico del contenido: ¿el paradigma perdido en la formación inicial y continua de profesores en Chile? Estudios pedagógicos (Valdivia), 40(número especial), 323-338. https://doi. org/10.4067/s0718-07052014000200019 
Vezub, L. (2016). Los saberes docentes en la formación inicial. La perspectiva de los formadores. Pensamiento Educativo, 53(1), 1-14. https://doi.org/10.7764/pel.53.1.2016.9 